\title{
Absence of capsular contracture in 319 consecutive augmentation mammaplasties: Dependent drains as a possible factor
}

\author{
Nabil Fanous MD FRCSC ${ }^{1}$, Iman Salem MD², Carolyne Tawilé MD FRCSC ${ }^{3}$, AE Bassas MD $^{4}$
}

N Fanous, I Salem, C Tawilé, AE Bassas. Absence of capsular contracture in 319 consecutive augmentation mammaplasties: Dependent drains as a possible factor. Can J Plast Surg 2004;12(4):193-197.

Capsular contracture is one of the major complications of augmentation mammaplasty. A review of 638 augmented breasts in 319 consecutive patients who underwent primary augmentation, with an average follow-up of 17.2 months and without a single case of capsular contracture of any degree to date, is presented, along with a discussion of the surgical technique and complications, and an analysis of measures used to prevent capsular contraction. Each patient received a pair of smooth saline-filled implants (Mentor, USA) placed in the submuscular space through an inframammary incision. In all operated breasts, many of the known measures commonly used for capsular contracture prevention were implemented. As well, a dependent drain was used as the final hemostatic step to prevent blood accumulation in the pocket. Leaving a dependent drain in the dissected pocket overnight, as one of the sequence of measures aimed at eliminating blood accumulation, is believed to be a contributing factor in capsular contracture prevention.

\section{Absence de contracture capsulaire dans 319 augmentations mammaires consécutives : Rôle possible des drains chirurgicaux}

Key Words: Augmentation mammaplasty; Drains; Prevention of capsular contraction

Capsular contracture is a particularly frustrating problem for Ueveryone concerned with breast augmentation: the surgeon, the patient and the manufacturer. It is virtually the single major complication of prosthetic use, yet has stubbornly resisted solutions despite more than 25 years of persistent clinical and laboratory investigation $(1,2)$.

Because sound etiological evidence has been lacking, many different theories have been advanced to explain capsular contracture: blood accumulation in the pocket secondary to hemorrhage, bacterial contamination, foreign body reaction to necrotic tissue, talcum powder or other irritants, gel bleed and/or inadequate dissection of the pocket.

The present report reviews 319 consecutive patients (638 breasts) who underwent primary augmentation mammaplasties with no incidence of capsular contracture to date. An analysis of the possible factors contributing to the absence, so far, of capsular contracture in this series is presented and compared with findings from previous studies on the subject.

\section{PATIENTS AND METHODS}

The present article is a retrospective study analyzing the incidence of capsular contracture in a series of primary bilateral esthetic augmentation mammaplasties. Bilateral augmentation mammaplasties were performed on 319 consecutive patients. All prostheses were placed in the submuscular plane through an inframammary incision. Each patient received a pair of smooth, saline-filled prostheses (style 1600, Mentor, USA). The implant size ranged from $150 \mathrm{~mL}$ to $425 \mathrm{~mL}$. The most commonly used size was $300 \mathrm{~mL}$ in 128 patients $(40.12 \%)$ followed by the $350 \mathrm{~mL}$ size implant in 69 patients $(21.63 \%)$.

\section{Preoperative evaluation}

Preoperatively, complete medical examinations and routine laboratory investigations were performed. Patients were asked to avoid smoking and to refrain from taking any acetylsalicylic acid derivatives or nonsteroidal anti-inflammatory drugs for at least two weeks before surgery.

*Montreal, Quebec. 'L'Institut Canadien de Chirurgie Esthétique and Department of surgery, Centre Hospitalier de LaChine, Montreal, Quebec;

${ }^{2}$ Department of Plastic Surgery, Alexandria University, Alexandria, Egypt (as a fellowship with Dr N Fanous); ${ }^{3}$ Department of Plastic Surgery,

Hôpital Charles Lemoyne, Montreal, Quebec; and ${ }^{4}$ Department of Plastic Surgery, Security Forces Hospital, Ryad, Saudi Arabia (as a fellowship with Dr N Fanous)

Correspondence: Dr N Fanous, 1 Westmount Square, Suite 1380, Westmount, Montreal, Quebec H3Z 2P9. Telephone 514-935-9906,

fax 514-935-6482, e-mail cosmeticsurgery123@videotron.ca 


\section{Preoperative marking}

The existing inframammary crease was marked while the patient was in a standing position. The incision was marked along the future inframammary crease and was centred on the breast meridian. To define the expected future shape and size of the breast, a tear-drop shaped pocket, with dimensions similar to or slightly larger than the base of the implant, was drawn on the skin. The perimeter of the marked pocket included a lateral tail-like extension of the dissection towards the axilla to simulate the natural breast configuration.

\section{Surgical technique}

In the present series, surgery was usually performed under local anesthesia with monitored sedation. Only 22 patients $(6.90 \%)$ had surgery under general anesthesia. The immediate preoperative routine included the administration of intravenous antibiotics (1 g Ancef, GlaxoSmithKline, USA) and steroids (250 mg Solu-Medrol, Pfizer, Canada). A tumescent solution, similar to the one commonly used in liposuction, was then used to infiltrate each breast pocket to anesthetize the surgical field, initiate submuscular elevation and help hemostasis. A totally submuscular pocket was elevated under three muscles - the pectoralis major, the serratus anterior and the external oblique muscles - by blunt dissection with instruments and without finger dissection. Cauterization was not commonly needed. If bleeding occured, it was usually minimal and easily cauterized in the subcutaneous or muscular levels. Routine irrigation of the pocket was performed with diluted Bacitracin (Pfizer, Canada) and Betadine (Purdue Pharma, Canada) solutions (and with only Bacitracin solution in the last 151 cases). The implant was then inserted and inflated using a closed system for sterile saline inflation to minimize the risk of contamination. In all patients, a very narrow dependent drain, in the form of a suction catheter or a feeding tube (size $8 \mathrm{Fr}, 2.7 \mathrm{~mm}$ ), was placed in the pocket, exiting at the lateral end of the incision, trimmed at approximately $5 \mathrm{~cm}$ out of the incision and fixed in place with adhesive tape (Steri-Strip, 3M, USA). The drain was usually removed $24 \mathrm{~h}$ postoperatively. The wound closure was done in two layers of muscle and dermis using $4-0$ or 3-0 vicryl (polyglactin 910) sutures. The patient left the recovery room approximately $2 \mathrm{~h}$ after surgery and was examined on the following day to check the breasts and remove the drains.

\section{Postoperative visits}

Following surgery, the patients were scheduled to return for visits after $24 \mathrm{~h}$, one week, one month, six months and then yearly from then on. The patients' compliance was enforced with an aggressive letter and telephone campaign to reach the patients and stress to them the importance of keeping their appointments.

On the first postoperative day, the drains were removed and general breast inspection was performed. During the one-week visit, the patients were instructed not to massage their breasts and not to apply heavy compression on them because of the authors' fear, although unproven, of possible implant displacement. The patients, however, were allowed to wear athletic bras or moderate elastic support for a few hours each day. On the subsequent visits, the patients were asked to assess their breast consistency. As well, the operating surgeon, and his assistant surgeon or clinic nurse, evaluated the breast consistency using the grading system proposed by Baker (3) and Spear and Baker (4).

\section{RESULTS}

A total of 638 operated breasts in 319 healthy female patients aged 18 to 56 years (average 29.6 years) were included in the
TABLE 1

Complications among 319 cases included in the study

\begin{tabular}{lll}
\hline Complication & $\mathbf{n}$ & $\%$ \\
\hline Capsular contracture (Baker II, III or IV) & 0 & 0.0 \\
Infection & 0 & 0.0 \\
Loss of nipple sensation & 0 & 0.0 \\
Hemorrhage & 1 & 0.31 \\
Temporary breast pain & 1 & 0.31 \\
Spontaneous deflation & 2 & 0.63 \\
Need for revision surgery & 4 & 1.25 \\
Hypertrophic scarring & 7 & 2.19 \\
\hline
\end{tabular}

present series. The follow-up ranged from six weeks to 45 months, with an average of 17.2 months. Follow-up ranged from one to approximately three years in 193 patients, between six months and one year in 82 patients, and the remaining 44 patients were followed for less than six months or over three years. It is important to note that only actual visits were considered in calculating the length of the follow-up; telephone or written communications were not considered because of their unreliability.

Overall, 25 patients (7.84\%) had other surgeries, such as liposuction, facial fat injections and rhinoplasties, performed at the same time as the augmentation mammaplasty procedure.

The patients were all satisfied with the softness of their breasts. All breasts were examined by the operating surgeon, as well as by another surgeon or nurse, during almost every visit and were found to be soft and were graded as Baker I. To date, there has not been a single incidence of Baker II, III or IV.

In terms of other complications (Table 1), there were no cases of infection. There was one case of immediate postoperative bleeding. This patient (an office staff member) developed a very slow but continuous dripping of blood from the drain of the right breast in the recovery room, approximately $30 \mathrm{~min}$ following surgery. The patient was returned to the operating room, where a deep muscular bleeder just inside the incision was easily cauterized. This patient has not developed capsular contracture for 26 months so far.

There were two incidences of spontaneous implant deflation three weeks and 12 months postoperatively that were reoperated and the prosthesis replaced.

Seven patients developed mild to moderate hypertrophic scarring, which improved with intralesional steroid injections and the use of silicone sheets for six to 18 months.

One patient had persistent pain in one breast, exaggerated by movement, for three months following surgery, which subsided gradually and spontaneously over a period of eight months.

No loss of nipple sensation was recorded. In one patient, the implant migrated inferiorly, and revision surgery was performed with no recurrence. Three patients had complaints about their breast volume; their implants were reoperated and the volume was adjusted. There were no cases of areolar necrosis, thrombophlebitis of chest wall veins (Mondor's disease) or pneumothorax.

\section{DISCUSSION}

Capsular contracture remains a major complication of aesthetic and reconstructive augmentation mammaplasty. The incidence of capsular contracture has been the subject of multiple 
studies with variable results (5-8). In a large study conducted by the Mentor manufacturer (5), the average rate of capsular contracture for saline-filled implants was $5 \%$ for a one-year follow-up and $9 \%$ for a three-year follow-up. Most of the capsular contracture cases tend to manifest themselves within the first year following surgery (9).

In spite of extensive clinical and basic science research, the etiology of this condition is still unresolved. As for any problem with an unknown etiology, the treatment remains speculative and multimodal. In the medical literature, the main hypotheses that attempt to explain the etiology of breast implant contracture are hematoma formation, bacterial infection, pocket placement, surface texture of the prosthesis, size of the implant, type of the implant filler, physiological response and lack of postoperative compression.

In our technique, we tried to consider a number of possible causes of capsular contracture and the corresponding measures suggested for their prevention, with a major emphasis on hemostasis; specifically, on preventing blood accumulation in the pocket.

The infectious theory assumes a subclinical infection with Staphylococcus epidermidis or other pathogens. Shah et al (10) and Netscher et al (11) demonstrated that inoculation with bacteria led to an increase in the thickness of capsules around implants. S epidermidis is known to live in the ductal system of the breast (12-14). It is also present in retromammary pockets before insertion of implants, as well as in established contractures (15). Finally, contamination of miniature mammary implants with $S$ epidermidis caused capsular contracture in experimental animals (10). Reduction of contracture was observed using intraoperative irrigation with $5 \%$ povidineiodine solution (16). Multiple publications $(1,17)$ have recommended intravenous intraoperative antibiotics and pocket irrigation with Bacitracin, Betadine or both. There is concern that agents used to disinfect the breast pockets have detrimental effects on wound healing $(18,19)$.

In our series, we used intraoperative antibiotics for systemic coverage as a single intravenous dose, and for pocket irrigation as a diluted solution.

Retromuscular implant placement, versus subglandular placement, has been shown in some studies (20-24) to decrease the incidence of contracture. There have been perceived problems in regard to subglandular placement, such as its possible association with capsular contracture (25). Biggs and Yarish (26) stated that the undesirable fibrous capsular contracture was significantly less frequent if implants were placed in the retropectoral space rather than the retroglandular one. Camirand et al (9) believed that the advantage in locating the implant behind the pectoral muscle is its efficient continuous compression and milking effect that reduces the spherical shape of the implant.

In our series, we used a completely submuscular implantation under three muscles, rather than the subglandular techniques.

Manual mobilization of the prosthesis (massage) is a very popular postoperative method for prevention of contracture. Camirand et al (9) stressed the importance of compressing the implant to increase its surface area, thereby stretching the periprosthetic contracting scar to prevent its contracture, and they instruct their patients to compress their implants for at least three months. Burkhardt (1), on the other hand, tried massage without success and doubts the effectiveness of intermittent positive compression.
In our postoperative instructions, we counselled our patients to refrain from any postoperative massage or severe compression for fear of the theoretical possibility of implant displacement. However, we allowed them to wear stretchy athletic bras or moderately elastic supports.

On the assumption that surface morphology is the prime factor in reducing capsular fibrosis, textured silicone-filled implants have been devised and used since 1988. Several reports (27-31) indicate that breasts with such implants are softer than those with smooth surfaced silicone prostheses.

Based on our results with only smooth implants, we doubt that textured implants are superior to the smooth-surfaced ones, and tend to agree with the findings of Tarpila et al (2) and others $(32,33)$, who concluded that there is no difference in contracture rates between textured and smooth implants. Baker (34) stated that the contracture rate of textured implants is equal to that of smooth-surfaced saline implants. Fagrell et al (7) showed no significant difference of contracture incidence of the smooth versus textured implants.

The use of silicone gel- versus saline-filled implants has been compared in the medical literature, with most data favouring the saline prosthesis as having a lower contracture rate $(35,36)$. In our practice, we use only saline-filled prostheses.

Regarding the size of the mammary prosthesis, Handel et al (8) found no demonstrable relationship between implant size and the ultimate likelihood of developing capsular contracture. However, there was a relationship between the implant size and the time course of contracture. In other words, the course of the capsular contracture was affected, but not its incidence. Analysis revealed that contracture occurred sooner with large (greater than $350 \mathrm{~mL}$ ) implants than with mediumor small-sized ones.

For purely aesthetic reasons, we do not usually use implants of more than $350 \mathrm{~mL}$. In our whole series, we used $375 \mathrm{~mL}$ implants in 42 patients and $425 \mathrm{~mL}$ implants in 27 .

Local steroids have been used in the past to suppress capsular contracture (37). Steroids were placed directly into the pocket or within an inflatable or double-lumen implant (38). Three-quarters of a series of patients treated by a technique that was recommended in 1976 later required the removal or the exchange of implants because of ptosis or severe soft tissue atrophy, and $13 \%$ of the same series still developed contracture despite the obviously high level of steroid administration $(38,39)$. Mladick $(40)$ also suggests that the use of steroid intraluminally or in pocket irrigation is not recommended.

In our series, we only used a single intraoperative intravenous dose of steroids for the purpose of decreasing postoperative edema. We did not use any steroidal preparations in the prosthesis or the pocket.

Finally, there is the hematoma theory. Hematoma, obvious or occult, was theoretically an attractive etiology because it could explain unilateral contracture (41). Handel et al (8) mentioned an increased risk of contracture in women with hematomas. They found that contracture occurred in 29\% of cases complicated by hematoma compared with $13 \%$ of cases without hematoma. Previously published studies (41-44) suggest that hematoma may be a predisposing factor to the development of capsular contracture. Goldberg (45) routinely used a $7 \mathrm{~mm}$ to $10 \mathrm{~mm}$ Jackson-Pratt drain for $24 \mathrm{~h}$ to $48 \mathrm{~h}$. The drain usually drained between $30 \mathrm{~mL}$ and $150 \mathrm{~mL}$ of serosanguinous fluid. In a routine augmentation mammaplasty, a meniscus of serosanguineous fluid may persist for weeks at 


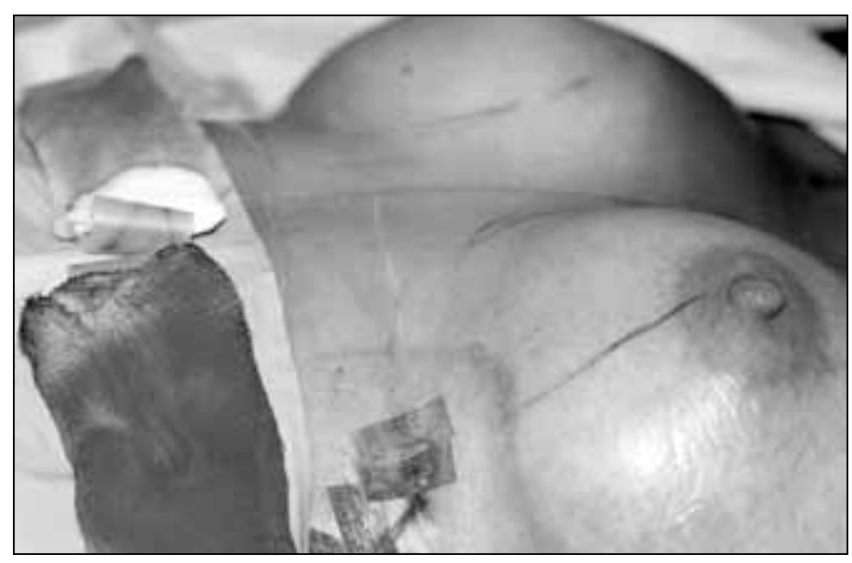

Figure 1) Example of a breast augmentation the morning following the procedure. The left drain is shown coming out of the lateral end of the inframmary incision, before being pulled out. The gauze dressings applied over the incisions at the time of the surgery are shown. The lighter looking dressing below the right breast is the typical one with pinkish serosanguinous liquid accumulation, while the darker dressing below the left breast shows a less frequent frankly red sanguinous drainage

the inferior pole of the periprosthetic space. This might initiate capsular contracture later on. The placement of the implant in the submuscular plane is thought to allow for persistent muscular tonus that may compress potential bleeders and, therefore, may lessen the chance of hematoma formation and subsequent contracture. As well, decreased vascularity of the submuscular plane, compared with the subglandular plane, may be another contributing factor to hemostasis.

We believe that prevention of hematoma formation is a major contributing factor in preventing subsequent capsular contracture. We implement preoperative, intraoperative and postoperative measures to help secure hemostasis and avoid blood accumulation. We insist that the patient stops using acetylsalicylic acid, acetylsalicylic acid derivatives, nonsteroidal anti-inflammatory drugs, herbal medicines and vitamins for a minimum of two weeks before surgery. Proper preoperative screening for hypertension is routinely done. Tumescent solution is used for local infiltration. A totally submuscular pocket is used. Completely blunt dissection of the pocket is practised in the great majority of cases. Detachment of the medial pectoralis is avoided. Fine cauterization of any bleeders, if present, is done.

As the final step to prevent blood accumulation in the pocket in case any of the previous precautions fails, a dependent drain is left in each breast pocket and is removed $24 \mathrm{~h}$ after surgery. Although most submuscular augmentations, in our experience, are quite dry at the end of the procedure, the drain usually brings out $10 \mathrm{~mL}$ to $50 \mathrm{~mL}$ of serosanguinous or frankly sanguinous fluid overnight (Figure 1). Removing this accumulation may be a reason behind the lack of capsular contracture. We did not try suction drains because of our early, very satisfactory experience with the dependent ones. In spite of the very small diameter $(2.7 \mathrm{~mm})$ of the drains we use, we find them to be highly efficient and practical. On the first postoperative day, pulling these drains out is completely painless. As well, during the first few seconds following their removal, a track is left behind and may be used to express further liquid if needed. The small opening at the incision site closes within minutes and usually leaves a fine scar.

\section{CONCLUSIONS}

The present report discusses 319 patients undergoing breast augmentation, with a follow-up ranging from six weeks to 45 months, with an average of 17.2 months and without a single case of capsular contracture of any degree to date. These early results are very encouraging, because the majority of capsular contracture cases tends to be detectable within the first postoperative year $(5,9)$.

In our technique, we implemented many of the known measures commonly used for prevention of capsular contracture. Intraoperative antibiotics were used for systematic coverage as a single intravenous dose, and for pocket irrigation as a diluted solution. Completely submuscular implantation was used, as opposed to the subglandular technique. Saline-filled prostheses were inserted. However, two known preventive measures - the performance of postoperative breast massage (or heavy compression) and the use of textured implants were not used by us, and therefore could not have had a positive impact on our results.

The main emphasis of our technique was the prevention of blood accumulation in the pocket postoperatively. Some of the hemostatic measures we used are popular, such as precise electrocauterization, tumescent fluid infiltration and completely blunt dissection. Of all the steps that are part of our inframammary augmentation technique, the one rarely used by other surgeons is the placement of drains (17), and especially dependent drains. We believe that drains give us our last chance at preventing blood accumulation in the pocket in case all previous steps fail. We consistently get drainage from these drains, usually in the form of a serous or serosanguineous liquid. However, this drainage is frankly sanguinous in approximately one of six breasts. If it were not for the drains, we believe this blood accumulation would go undetected because of its relatively limited volume.

We believe that leaving a drain in the dissected pocket is an important contribution to the sequence of steps aiming at eliminating blood accumulation, and is a contributing factor in preventing capsular contracture in augmentation mammaplasty.

ACKNOWLEDGEMENTS: The authors express their thanks to Robert Derval, medical photographer, for his photographic contribution; to Barbara Armbruster, MA for the editing; to Derya Atilgan for the typing; and to Amanda Fanous for the medical literature search.

\section{REFERENCES}

1. Rohrich RJ, Kenkel JM, Adams WP. Preventing capsular contracture in breast augmentation: In search of the Holy Grail. Plast Reconstr Surg 1999;103:1759-60; discussion 1769-72.

2. Tarpila E, Ghassemifar R, Fagrell D, Berggren A. Capsular contracture with textured versus smooth saline-filled implants for breast augmentation: A prospective clinical study. Plast Reconstr Surg 1997;99:1934-9.

3. Baker J. Classification of spherical contractures. Presented at the Aesthetic Breast Symposium, Scottsdale, Arizona, 1975.

4. Spear SL, Baker JL Jr. Classification of capsular contracture after prosthetic breast reconstruction. Plast Reconstr Surg 1995;96:1119-23.

5. Mentor Inc. Saline-filled breast implant surgery: Making an informed decision <http://www.mentorcorp.com/pdf/FinalInformedConsent.pdf $>$ Updated January 2004. (Version current at September 30, 2004)

6. Gutowski KA, Mesna GT, Cunningham BL. Saline-filled breast implants: A Plastic Surgery Educational Foundation multicenter outcomes study. Plast Reconstr Surg 1997;100:1019-27.

7. Fagrell D, Berggren A, Tarpila E. Capsular contracture around salinefilled fine textured and smooth mammary implants: A prospective 7.5-year follow-up. Plast Reconstr Surg 2001;108:2108-12. 
8. Handel N, Jensen JA, Black Q, Waisman JR, Silverstein MJ. The fate of breast implants: A critical analysis of complications and outcomes. Plast Reconstr Surg 1995;96:1521-33.

9. Camirand A, Doucet J, Harris J. Breast augmentation: Compression a very important factor in preventing capsular contracture. Plast Reconstr Surg 1999;104:529-38.

10. Shah Z, Lehman JA, Tan J. Does infection play a role in breast capsular contracture? Plast Reconstr Surg 1981;68:34-42.

11. Netscher DT, Weizer G, Wigoda P, Walker LE, Thornby J, Bowen D. Clinical relevance of positive breast periprosthetic cultures without overt infection. Plast Reconstr Surg 1995;96:1125-9.

12. Argenta L, Grabb W. Studies on the endogenous flora of the human breast and their surgical significance. Presented at the Annual Meeting of the American Society of Plastic \& Reconstructive Surgeons, New York City, New York, October 20, 1981.

13. Boer HR, Anido G, MacDonald N. Bacterial colonization of human milk. South Med J 1981;74:716-8

14. Courtiss EH, Goldwyn RM, Anastasi GW. The fate of breast implants with infections around them. Plast Reconstr Surg $1979 ; 63: 812-6$

15. Burkhardt BR, Fried M, Schnur PL, Tofield JJ. Capsules, infection and intraluminal antibiotics. Plast Reconstr Surg 1981;68:43-9.

16. Kwaan JH, Connolly JE. Successful management of prosthetic graft infection with continuous povidone-iodine irrigation. Arch Surg 1981;116:716-20.

17. Becker H, Springer R. Prevention of capsular contracture. Plast Reconstr Surg 1999;103:1766-8; discussion 1773-4

18. Lineaweaver W, Howard R, Soucy D, et al. Topical antimicrobial toxicity. Arch Surg 1985;120:267-70.

19. Lord JW Jr, LaRaja RD, Daliana M, Gordon MT. Prophylactic antibiotic wound irrigation in gastric, biliary and colonic surgery. Am J Surg 1983;145:209-12.

20. Jarrett JR, Certler RG, Teal DF. Subcutaneous mastectomy in small, large, or ptotic breasts with immediate submuscular placement of the implants. Plast Reconstr Surg 1978;62:702-5.

21. Mahler D, Hauben DJ. Retromammary versus retropectoral breast augmentation: A comparative study. Ann Plast Surg 1982;8:370-4.

22. Woods JE, Irons GB, Arnold PG. The case for submuscular implantation of prostheses in reconstructive breast surgery. Ann Plast Surg 1980;5:115-22.

23. Slade CL. Subcutaneous mastectomy: Acute complications and long-term follow-up. Plast Reconstr Surg 1984;73:84-90.

24. Tebbets JB. Transaxillary subpectoral augmentation mammaplasty: Long-term follow up and refinements. Plast Reconstr Surg 1984;74:636-49.

25. Sohn BK, Chung YJ, Kim G, Yoon WJ. Submuscular periareolar approach to augmentation mammaplasty in Korean women. Aesthetic Plast Surg 2000;24:455-60.

26. Biggs TM, Yarish RS. Augmentation mammaplasty: A comparative analysis. Plast Reconstr Surg 1990;85:368-72.
27. Coleman DJ, Foo ITH, Sharpe DT. Textured or smooth implants for breast augmentation? A prospective, controlled trial. Br J Plast Surg 1991;44:444-8.

28. Hakelius L, Ohlsen L. A clinical comparison of the tendency to capsular contracture between smooth and textured gel-filled mammary implants. Plast Reconstr Surg 1992;90:247-54.

29. Asplund O, Gylbert L, Jurell G, Ward C. Textured or smooth implants for submuscular augmentation: A controlled study. Plast Reconstr Surg 1996;97:1200-6.

30. Ersek RA. Rate and incidence of capsular contracture: A comparison of smooth and textured silicone double-lumen breast prostheses. Plast Reconstr Surg 1991;87:879-84.

31. Pollock H. Breast capsular contracture: A retrospective study of textured versus smooth silicone implants. Plast Reconstr Surg 1993;91:404-7.

32. Shapiro MA. Smooth vs. rough: An 8-year survey of mammary prostheses. Plast Reconstr Surg 1989;84:449-57.

33. Copeland M, Choi M, Bleiweiss IJ. Silicone breakdown and capsular synovial metaplasia in textured-wall saline breast prostheses. Plast Reconstr Surg 1994;94:628-33.

34. Biggs TM. Augmentation mammaplasty: A comparative analysis. Plast Reconstr Surg 1999;103:1761-2; discussion 1763-5.

35. Gylbert L, Asplund O, Jurell G. Capsular contracture after breast reconstruction with silicone gel and saline filled implants: A 6-year follow-up. Plast Reconstr Surg 1990;85:373-7.

36. Cairns TS, De Villiers W. Capsular contracture after breast augmentation - a comparison between gel- and saline-filled prostheses. S Afr Med J 1980;57:951-3.

37. Peterson HD, Burt GB Jr. The role of steroids in prevention of circumferential capsular scarring in augmentation mammaplasty. Plast Reconstr Surg 1974;54:28-30.

38. Perrin ER. The use of soluble steroids within inflatable breast prostheses. Plast Reconstr Surg 1976;57:163-6.

39. Oneal RM, Argenta LC. Late side effects related to inflatable breast prostheses containing soluble steroids. Plast Reconstr Surg 1982;69:641-5.

40. Mladick RA. "No-touch" submuscular breast augmentation technique. Aesthetic Plast Surg 1993;17:183-92.

41. Williams C, Aston S, Rees T. The effect of hematoma on the thickness of pseudo sheaths around silicone implants. Plast Reconstr Surg 1975;56:194-8.

42. Hipps CJ, Raju DR, Straith RE. Influence of some operative and postoperative factors on capsular contracture around breast prostheses. Plast Reconstr Surg 1978;61:384-9.

43. Myers B, Rightor M. Augmentation of wound tensile strength in rats by induction of inflammation with autogenous blood. Surgery 1978;83:78-82

44. Watson J. Some observations on breast augmentation procedures over the past two decades. Aesthetic Plast Surg 1976;1:89.

45. Goldberg HM. Drains and breast implants. Plast Reconstr Surg 1988;81:990 